\title{
High Grade Salivary Gland Mucoepidermoid Carcinoma
}

National Cancer Institute

\section{Source}

National Cancer Institute. High Grade Salivary Gland Mucoepidermoid Carcinoma. NCI

Thesaurus. Code C8019.

A salivary gland mucoepidermoid carcinoma with high grade histopathologic features. It usually follows an aggressive clinical course. 